\title{
Anatomical localization of insulinomas: still a need to combine a set of diagnostic procedures
}

\author{
Gianluca Tamagno, Donal O’Shea
}

Department of Endocrinology \& Diabetes Mellitus, St Vincent's University Hospital - University College Dublin, Ireland

\begin{abstract}
The diagnosis of insulinoma is based on universally defined clinical and laboratory parameters (Whipple triad and fasting test). Pre-operative tumor localization is the main challenge in the diagnostic management of insulinomas. The pre-operative and/or intra-operative localization of the tumor is required for the optimal surgical approach. We describe two cases of insulinoma characterized by a typical clinical presentation, a positive diagnosis on fasting test, and computerized tomography failure in localizing the tumor. In the first patient, angiography with hepatic venous sampling after calcium stimulation correctly localized pre-operatively the region of the pancreas where the tumor was and, following intra-operative investigations, the lesion was successfully enucleated. In the second patient, angiography with hepatic venous sampling after calcium stimulation failed to identify the region of the insulinoma, which was detected by intra-operative ultrasound and successfully enucleated. Invasive pre-operative procedures for tumor regionalization and/or localization (angiography with hepatic venous sampling after calcium stimulation, endoscopic ultrasound) should be performed in cases where the tumor cannot be localized with enough certainty by non-invasive imaging. However, a careful intra-operative study should be performed in all patients undergoing surgery to complete the information obtained pre-operatively and to exclude the presence of other smaller lesions.
\end{abstract}

Key words: Calcium stimulation, Endoscopic ultrasound, Hepatic venous sampling, Insulinoma, Neuroendocrine tumor

\section{INTRODUCTION}

Insulinoma represents the most common functioning endocrine tumor of the pancreas with an estimated incidence of 1-3 per million per year. ${ }^{1}$ Often, these

\section{Address for correspondence:}

Dr Gianluca Tamagno, Department of Endocrinology and Diabetes Mellitus, St Vincent's University Hospital University College Dublin, 4 Elm Park, Dublin 4, Ireland, Tel.: +353 1221 4407, E-mail: gianlucatamagno@tiscali.it Received 10-01-12, Revised 27-03-12, Accepted 11-05-12 tumors are benign or of uncertain malignant potential but occasionally they are definitely malignant. ${ }^{2}$ The clinical manifestations may range from dramatic autonomic nervous system and/or neuroglycopenic symptoms to more subtle presentations. Diagnosis of insulinoma is based on defined clinical and laboratory parameters derived from the Whipple triad (hypoglycemia symptoms in the presence of low plasma glucose with clinical relief on glucose administration) and the prolonged fasting test. The macroscopic, microscopic, 
and immunohistochemical features required for the diagnosis of insulinoma have been defined by the current World Health Organization classification of the neuroendocrine pancreatic tumors. ${ }^{1,2}$ While the anatomical aspects and the functional behavior can cause pitfalls in the detection of some insulinomas, ${ }^{3}$ pre-operative tumor characterization and localization is the main challenge in the diagnostic management of these lesions. A set of non-invasive and invasive preoperative imaging procedures can be used to localize the lesion ${ }^{4}$ but sometimes the definitive anatomical identification is obtained only intra-operatively. Although angiography with hepatic venous sampling after calcium stimulation was previously considered the gold standard for the regionalization of insulinoma, today this is mostly reserved for problematic cases. ${ }^{1}$ However, this test remains a very sensitive tool for the regionalization of insulinomas in centers with experienced personnel ${ }^{5-7}$ and its effectiveness remains high even when low doses of calcium are injected in order to minimize the procedural risks. ${ }^{8}$ We describe two illustrative cases of insulinoma in which the correct localization of the tumor required pre-operative and intra-operative procedures and was achieved by different modalities.

\section{CASE REPORTS}

\section{Case 1}

A 38-year-old woman presented with a 12-month history of disorientation on waking resolved by eating ( 1 episode/2 months on average), increased appetite, and weight gain $(6 \mathrm{~kg})$. Her past medical history was unremarkable and she was not taking any medication. She had persistent morning capillary hypoglycemia (1.6 - $2.9 \mathrm{mmol} / \mathrm{L})$. Her routine biochemical profile was normal and the sulphonylurea screening was negative. Diagnosis of insulinoma was confirmed by a 72 -hour fasting test (at hour 13, venous glucose $1.7 \mathrm{mmol} / \mathrm{L}$, insulin $7.4 \mathrm{mU} / \mathrm{L}$, and C-peptide 3.0 $\mathrm{mcg} / \mathrm{L}$ - reference range: 0.2 - 3.2). She commenced diazoxide, titrating the dose up to $100 \mathrm{mg}$ twice daily. Computerized tomography failed to identify and localize a lesion. Both angiography with hepatic venous sampling after calcium stimulation (Table 1a) and endoscopic ultrasound localized the tumor at the pancreatic head and the patient underwent
Table 1a. Insulin levels (mU/L) at angiography with hepatic venous sampling after arterial calcium stimulation in a patient with insulinoma localized in the area of pertinence of the gastroduodenal artery, corresponding to the pancreatic head

\begin{tabular}{lccccc}
\hline & HA & GDA & SMA & PSA & DSA \\
\hline t -120 & 14.4 & 23.4 & 21.4 & 18.1 & 16.0 \\
t 0 & 18.8 & 21.0 & 17.9 & 16.3 & 16.5 \\
t 30 & 17.0 & 124.7 & 16.5 & 20.1 & 18.1 \\
t 60 & 16.3 & 229.6 & 17.3 & 25.8 & 17.3 \\
t 90 & 19.0 & 240.3 & 19.1 & 21.9 & 18.1 \\
t 120 & 20.5 & 230.6 & 18.7 & 23.1 & 18.4 \\
t 180 & 19.4 & 140.0 & 15.7 & 18.4 & 18.6 \\
\hline
\end{tabular}

HA: hepatic artery; GDA: gastroduodenal artery; SMA: superior mesenteric artery; PSA: proximal splenic artery; DSA: distal splenic artery. Time (t): seconds.

laparotomy. Exploration of the pancreas and intraoperative ultrasound confirmed the presence of a single $1.2 \mathrm{~cm}$ tumor localized in the pancreatic head adjacent to the portal vein and did not reveal any further areas of tumor involvement. The tumor was successfully enucleated with an unremarkable postoperative course. Post-operative fasting glucose was $5.4 \mathrm{mmol} / \mathrm{L}$ (day 1). Conventional histological examination showed a well circumscribed lesion composed of neuroendocrine cells with minimal atypia and no mitotic activity or necrosis, corresponding to a well-differentiated endocrine tumor (WHO 1). Immunohistochemistry was positive for synaptophysin and weakly positive for chromogranin A, while insulin immunostaining was negative.

\section{Case 2}

A 54-year-old woman presented with a 6-month history of confusion, behavioral abnormalities, and involuntary movements associated with fasting (8 episodes/month on average). Her past medical history was unremarkable and she was not taking any medication. She had persistent morning capillary hypoglycemia $(1.6-2.9 \mathrm{mmol} / \mathrm{L})$. Her routine biochemical profile was normal and the sulphonylurea screening was negative. Diagnosis of insulinoma was confirmed by a 72 -hour fasting test (at hour 3 , venous glucose $1.9 \mathrm{mmol} / \mathrm{L}$, insulin $15.2 \mathrm{mU} / \mathrm{L}$, and C-peptide $2.2 \mathrm{mcg} / \mathrm{L}$ - reference range: 0.2 - 3.2). She started treatment with diazoxide, titrating the dose up to 200 $\mathrm{mg}$ three times a day due to recurrent hypoglycaemic 
episodes, but the tolerance to the diazoxide treatment was poor. A calcium channel blocker was added. The computerized tomography scan was inconclusive and angiography with hepatic venous sampling after calcium stimulation (Table 1b) failed to define the region of the lesion on two occasions. Endoscopic ultrasound was not done. The patient underwent laparotomy for surgical exploration. Pancreatic palpation was inconclusive but a nodule with typical features of islet cell tumor at the lower border of the body of the pancreas was identified by intra-operative ultrasound and successfully enucleated (size $1.5 \times 1 \times 0.5$ $\mathrm{cm})$. Post-operative fasting glucose was $5.7 \mathrm{mmol} / \mathrm{L}$ (day 1) and the patient remained stable after surgery. Conventional histological examination showed a welldifferentiated neuroendocrine tumor with an insular growth pattern (WHO 1). Mitotic figures were not identified. At immunohistochemistry, the tumor was strongly positive for both synaptophysin and insulin.

\section{DISCUSSION}

These two clinical cases demonstrate different aspects of the typical clinical presentation and the localization issues associated with the diagnosis of insulinoma. Conventional, non-invasive radiological methods failed to localize the tumor in both patients and an invasive pre-operative study was necessary. The angiography with hepatic venous sampling after calcium stimulation successfully identified the region where the insulinoma was located in one of the patients

Table 1b. Insulin levels (mU/L) at angiography with hepatic venous sampling after arterial calcium stimulation in a patient with insulinoma which was not localized anatomically

\begin{tabular}{lcccccc}
\hline & RHA & LHA & GDA & SMA & PSA & DSA \\
\hline t -120 & 30.1 & 25.4 & 11.3 & 38.6 & 11.9 & 5.9 \\
t 0 & 27.3 & 22.6 & 24.1 & 32.7 & 12.1 & 8.1 \\
t 30 & 24.2 & 22.3 & 26.1 & 32.8 & 16.3 & 8.7 \\
t 60 & 29.6 & 16.5 & 31.2 & 34.9 & 14.9 & 10.7 \\
t 90 & 29.6 & 19.7 & 26.4 & 39.4 & 16.2 & 11.1 \\
t 120 & 36.8 & 19.6 & 23.5 & 36.4 & 14.6 & 10.3 \\
t 180 & 33.2 & 17.2 & 24.7 & 41.6 & 17.5 & 12.7 \\
\hline
\end{tabular}

RHA: right hepatic artery; LHA: left hepatic artery; GDA: gastroduodenal artery; SMA: superior mesenteric artery; PSA: proximal splenic artery; DSA: distal splenic artery. Time (t): seconds. by showing an increase in hepatic venous insulin concentration after the arterial calcium injection equal to or greater than 2-fold from baseline. ${ }^{5,7}$ An integrated pre-operative and intra-operative diagnostic study for tumor localization was properly done and finally allowed the anatomical definition of the lesion in both cases, as required for the most appropriate, safe, and effective surgical approach..$^{9-11}$ The histological examination of the surgical specimens showed definite neuroendocrine features that were compatible with the diagnosis of insulinoma in both patients when combined with the clinical manifestations and the biochemical data. One of the two insulinomas was negative for insulin immunostaining, an occasional finding in these lesions, which may result from a rapid release of insulin by tumor insulin-secreting cells with a failure to store.

Overall, the findings described in these two patients demonstrate that a set of complementary noninvasive and invasive imaging techniques (including intra-operative assessment) is helpful in the correct localization of insulinoma. The choice of the radiological procedures in the localization algorithm for an insulinoma patient should be optimized to allow the detection of the tumor in the least invasive and most sensitive manner. This can assist the surgeon in the most effective and minimally invasive therapeutic approach in theatre. ${ }^{12}$ In the modern era, the pre- and intra-operative diagnostic tools make the successful localization of insulinomas an achievable goal in centers with wide and longstanding experience. ${ }^{13,14}$ The application of new protocols and techniques is improving the sensitivity of magnetic resonance imaging for the identification of insulinomas. ${ }^{15}$ Also contrast-enhanced ultrasound may work as an effective and non-invasive localization modality and presents the advantage of being inexpensive. ${ }^{16}$ If a pre-operative invasive radiological investigation is needed, the choice of angiography with hepatic venous sampling after calcium stimulation versus endoscopic ultrasound is driven by the availability of the procedure and the experience of the investigators. No studies aiming to compare the sensitivity of these two techniques for insulinoma localization have been carried out. Both procedures can be considered as highly and comparably sensitive for this purpose..$^{5,7,17,18}$ However, endoscopic ultrasound is progressively overtaking 
angiography with hepatic venous sampling after calcium stimulation as it can provide a more sensitive identification of the tumor localization and may help with the detection of multiple lesions or the definition of the most suitable, effective, and pancreatic parenchyma-preserving surgical approach, including minimally invasive surgery. ${ }^{19}$ For instance, endoscopic ultrasound-guided tattooing of insulinoma has been suggested as a very helpful pre-operative technique which may facilitate the laparoscopic enucleation of the tumor. ${ }^{20,21}$

The two illustrative cases above described confirm that the diagnosis of insulinoma is based on the clinical presentation, using the Whipple triad and the prolonged fasting test. The optimal therapeutic approach to insulinoma requires pre-operative anatomical detection by conventional, non-invasive radiological methods and, in cases where the noninvasive radiological tools fail in localizing the tumor, invasive procedures such as angiography with hepatic venous sampling after calcium stimulation and/or endoscopic ultrasound should be used. Ultimately, intra-operative localization by pancreatic palpation and intra-operative ultrasound may be required. However, the intra-operative study should be carefully performed in all patients undergoing surgery to confirm the information obtained through preoperative characterization of the tumor and to exclude the presence of other smaller lesions. Angiography with hepatic venous sampling after calcium stimulation still represents a useful procedure for tumor localization in selected cases. However, it may fail due to technical or anatomical reasons and its value is limited only to the regionalization of the lesion. In our opinion, invasive pre-operative procedures for insulinoma localization (angiography with hepatic venous sampling after calcium stimulation and endoscopic ultrasound) provide complementary information and both investigations should be performed in cases where the tumor cannot be localized with enough certainty by a single test alone.

\section{The authors did not receive any grant or fellowship to support this work}

\section{REFERENCES}

1. de Herder WW, Niederle B, Scoazec JY, et al, 2006 Well- differentiated pancreatic tumor/carcinoma: insulinoma. Neuroendocrinology 84: 183-188.

2. Rindi G, Kloppel G, 2004 Endocrine tumors of the gut and pancreas tumor biology and classification. Neuroendocrinology 80: Suppl 1: 12-15.

3. Tamagno G, Maffei P, Pasquali C, et al, 2005 Clinical and diagnostic aspects of cystic insulinoma. Scand J Gastroenterol 40: 1497-1501.

4. Jensen RT, Cadiot G, Brandi ML, et al, 2012 ENETS consensus guidelines for the management of patients with digestive neuroendocrine neoplasms: functional pancreatic endocrine tumor syndromes. Neuroendocrinology 95: 98-119.

5. Doppman JL, Chang R, Fraker DL, et al, 1995 Localization of insulinomas to regions of the pancreas by intra-arterial stimulation with calcium. Ann Intern Med 123: $269-273$.

6. Goh BK, Ooi LL, Cheow PC, et al, 2009 Accurate preoperative localization of insulinomas avoids the need for blind resection and reoperation: analysis of a single institution experience with 17 surgically treated tumors over 19 years. J Gastrointest Surg 13: 1071-1077.

7. Guettier JM, Kam A, Chang R, et al, 2009 Localization of insulinomas to regions of the pancreas by intraarterial calcium stimulation: the NIH experience. J Clin Endocrinol Metab 94: 1074-1080.

8. O'Shea D, Rohrer-Theurs AW, Lynn JA, Jackson JE, Bloom SR, 1996 Localization of insulinomas by selective intraarterial calcium injection. J Clin Endocrinol Metab 81: 1623-1627.

9. Geoghegan JG, Jackson JE, Lewis MP, et al, 1994 Localization and surgical management of insulinoma. $\mathrm{Br}$ J Surg 81: 1025-1028.

10. Roland CL, Lo CY, Miller BS, Holt S, Nwariaku FE, 2008 Surgical approach and perioperative complications determine short-term outcomes in patients with insulinoma: results of a bi-institutional study. Ann Surg Oncol 15: 3532-3537.

11. Isla A, Arbuckle JD, Kekis PB, et al, 2009 Laparoscopic management of insulinomas. Br J Surg 96: 185-190.

12. Rostambeigi N, Thompson GB, 2009 What should be done in an operating room when an insulinoma cannot be found? Clin Endocrinol (Oxf) 70: 512-515.

13. Placzkowski KA, Vella A, Thompson GB, et al, 2009 Secular trends in the presentation and management of functioning insulinoma at the Mayo Clinic, 1987-2007. J Clin Endocrinol Metab 94: 1069-1073.

14. Nikfarjam M, Warshaw AL, Axelrod L, et al, 2008 Improved contemporary surgical management of insulinomas: a 25-year experience at the Massachusetts General Hospital. Ann Surg 247: 165-172.

15. Druce MR, Muthuppalaniappan VM, O'Leary B, et al, 2010 Diagnosis and localisation of insulinoma: the value of modern magnetic resonance imaging in conjunction with calcium stimulation catheterization. Eur J Endocrinol 162: 971-978. 
16. An L, Li W, Yao KC, et al, 2011 Assessment of contrastenhanced ultrasonography in diagnosis and preoperative localization of insulinoma. Eur J Radiol 80: 675-680.

17. Rösch T, Lightdale CJ, Botet JF, et al, 1992 Localization of pancreatic endocrine tumors by endoscopic ultrasonography. N Engl J Med 326: 1721-1726.

18. McLean A, 2004 Endoscopic ultrasound in the detection of pancreatic islet cell tumours. Cancer Imaging 4: 84-91.

19. Vaidakis D, Karoubalis J, Pappa T, Piaditis G, Zografos
GN, 2010 Pancreatic insulinoma: current issues and trends. Hepatobiliary Pancreat Dis Int 9: 234-241.

20. Gress FG, Barawi M, Kim D, Grendell JH, 2002 Preoperative localization of a neuroendocrine tumor of the pancreas with EUS-guided fine needle tattooing. Gastrointest Endosc 55: 594-597.

21. Zografos GN, Vasiliadis G, Karoubalis J, Tsagarakis S, 2010 Laparoscopic resection of insulinoma after endoscopic ultrasonography tattooing. Am Surg 76: 446-448. 